\title{
Activation of mek alters morphology and neuroendocrine phenotype in medullary thyroid cancer cell
}

\begin{abstract}
Background: Medullary thyroid cancer (MTC) is a neuroendocrine (NE) tumor of the thyroid C cells. Surgery is the only curative therapy. Activation of raf- 1 in MTC cells resulted in growth suppression and NE marker reduction. However, the exact mediator of this effect is not clearly understood. We hypothesize that MEK, a key downstream target of raf-1 pathway, may be involved in the effect seen with raf-1 activation in MTC. To determine the role of MEK, we established a doxycycline-inducible MEK in MTC TT cells and assessed the effects on morphology and NE phenotype.
\end{abstract}

Methods: Doxycycline-inducible TT-MEK cells were created by stable transfection of a pRevTRE-MEK plasmid in TT cells expressing a Tet responsive protein. TT-MEK cells were treated with $0-1.0 \mu \mathrm{g} / \mathrm{ml}$ of doxycycline for four days. The level of total and active MEK expression was determined by western blot analysis using MEK and phosphorylated ERK1/2 antibodies. In addition, the lysates were analyzed for levels of the NE markers achaete-scute complex like 1 (ASCL1) and Chromogranin A (CgA). Morphology of the treated and control cells was observed under a light microscope.

Results: Treatment of TT-MEK cells with doxycycline led to an induction of MEK protein, which is associated with activation of ERK1/2 in a dose-dependent manner. Importantly, the levels of NE markers were reduced by increasing MEK activation. Similar to RAF-1 activation, there was a striking morphological change with activation of MEK1/2, with a decrease in horizontal spread of each cell resulting in rounder, larger cells.

Conclusion: We demonstrate, for the first time, that the over expression of MEK in MTC cells resulted in similar effects of raf-1 pathway activation. Thus, MEK may serve as a molecular target and a therapeutic strategy to treat patients with MTC.

Keywords: medullary thyroid cancer, neuroendocrine, thyroid gland, carcinoid cells, radiotherapy, chemotherapy, tt-mek cells, penicillin, streptomycin, hormones
Volume 3 Issue 4 - 2016

\section{Amal Alhefdhi,' Muthusamy Kunnimalaiyaan, ${ }^{2}$ Herbert Chen ${ }^{3}$ \\ 'Department of Surgery, King Faisal Specialist Hospital and Research Centre, Al Faisal University, Saudi Arabia \\ ${ }^{2}$ Department of Surgery, Medical College of Wisconsin, USA \\ ${ }^{3}$ Department of Surgery, University of Alabama at Birmingham (UAB), UK}

Correspondence: Herbert Chen, Professor and Chairman, Department of Surgery, University of Alabama at Birmingham (UAB), Surgeon-in-Chief, UAB Health System, Boshell Building, Suite 502, UK, Tel + I 205934 3333, Email herbchen@uab.edu

Received: September 08, 2016 | Published: November 15, 2016
Abbrevations: MTC, medullary thyroid cancer; CgA, chromogranin A; ASCL1, achaete scute complex-like 1; ASH1, achaete scute homolog-1; MAPKs, mitogen-activated protein kinases; ERK1, extracellular signal-regulated kinases; VEGFR, vascular endothelial growth factor receptor; MEK, mitogen-activated protein kinase; ERK, extracellular signal-regulated kinase; NE, neuro endocrine

\section{Synopsis}

Activation of the MEK pathway in MTC cells elicited similar effects of raf-1 pathway activation, resulting in cell growth suppression. Therefore, MEK may serve as a molecular target and a therapeutic strategy to treat patients with MTC.

\section{Introduction}

Medullary thyroid cancer (MTC) is a neuroendocrine (NE) tumor that arises from the parafollicular cells (C-cells) of the thyroid gland. ${ }^{1}$ MTC accounts for $5-10 \%$ of all thyroid malignancies and responds poorly to chemotherapy. ${ }^{2}$ Thus, the only curative option for MTC is surgical resection. ${ }^{1}$ MTC is the end result of an oncogenic activation of protein cascades in the NE cells of the thyroid gland. ${ }^{3}$ Hence, understanding the molecular pathways that control C-cell proliferation is vital for developing novel therapies for patients with advanced MTC. ${ }^{4}$

MTC cells produce several hormones and NE markers such as calcitonin, Chromogranin A (CgA),${ }^{5}$ and Achaete Scute ComplexLike 1 (ASCL1), also known as human ASH1 (Achaete Scute Homolog-1) ${ }^{6,7}$ ASCL1 is involved in C-cell expansion and is critical for neuroendocrine cell differentiation and MTC tumor growth. The importance of ASCL1 for C-cell growth was observed in transgenic mice with an ASCL1 knockout gene, which failed to develop thyroid C-cells. ${ }^{1}$ Moreover, ASCL1 has been found to be involved in the development of other NE cells such as pulmonary endocrine cells and chromaffin cells. ${ }^{1}$

Sustained Raf-1 activation in MTC cells leads to reduction in ASCL1, CgA, and consequently cell growth arrest. ${ }^{8}$ Mitogenactivated protein kinases (MAPKs) represent the largest family of protein kinases that control cellular proliferation, cell differentiation and apoptosis by regulating diverse intracellular signal transduction cascades. MAPKs fall into four categories: the extracellular signal- 
regulated kinases (ERK1 and ERK2), c-Jun NH2- terminal/stressactivated protein kinases, p38 MAPKs, and ERK5. ${ }^{9}$

Raf-1 acts as a kinase repressor in MAPKs and its activation in MTC cells results in growth arrest and ultimately a reduction in NE marker production. ${ }^{10,11}$ However, the exact mediator of this effect is not clearly understood. In this study we hypothesize that MEK, a key downstream target of the raf-1 pathway, may be involved in the effect seen with raf-1 activation in MTC. To determine the effect of MEK in NE tumors, we established a doxycycline-inducible MEK in MTC TT cells and assessed the effects on morphology and NE phenotype.

\section{Materials and methods}

\section{Cell culture}

Human MTC TT cells were obtained from Dr. Barry D. Nelkin (The Johns Hopkins University, Baltimore, MD) and maintained in RPMI 1640 medium (Invitrogen) supplemented with 18\% fetal bovine serum (Sigma), $100 \mathrm{IU} / \mathrm{ml}$ penicillin (Invitrogen), and 100mg/ $\mathrm{ml}$ streptomycin (Invitrogen) in a humidified atmosphere of 5\% $\mathrm{CO}_{2}$ in the air at $37^{\circ} \mathrm{C} .{ }^{1,10}$ Doxycycline-inducible cell lines were maintained similar to TT cells except that tetracycline-free fetal bovine serum (Clontech), $0.4 \mathrm{mcg} / \mathrm{ml} \mathrm{G} 418$ (Invitrogen), and $0.4 \mathrm{mcg} / \mathrm{ml}$ hygromycin (Invitrogen) were used in the cell medium.

\section{Doxycycline-inducible MEK}

The Tet-On expression system was obtained from Clontech. A BamHI-XhoI fragment containing MEK plasmid was sub cloned into the pRevTRE vector at the BamHI/Sall sites. To create an inducible TT-MEK cell line, TT cells were first transfected with plasmid pRevTet-On (Clontech) containing the Tet-responsive transcriptional activator using Lipofectamine 2000 (Invitrogen) and selected in medium containing $0.4 \mathrm{~g} / \mathrm{ml} \mathrm{G} 418 .{ }^{1}$ The resulting G418resistant, TT-Tet-on clone was screened for doxycycline-dependent inducibility of the reporter gene luciferase by transient transfection of the pRevTRE-Luc vector. ${ }^{1}$ Low background was maintained and used for transfection with the response plasmid containing the MEK gene (pRevTRE-MEK) and the empty vector (pRevTRE). TT-Tet-on cells were transfected with pRevTRE-MEK and the pRevTRE empty vector using lipofectamine.

\section{Induction}

MTC-TT cells were treated with doxycycline for up to 4 days using different doses $(01 \mu \mathrm{g} / \mathrm{ml})$.

\section{Western blot analysis}

Cell pellets were lysed in sample buffer as previously described..$^{1,8}$ Total cellular protein concentrations were determined using a BCA assay kit (Pierce) according to manufacturer's instructions. Cell extracts $(20-30 \mu \mathrm{g})$ were boiled with equal amounts of loading dye for $10 \mathrm{~min}$ and separated on $10 \%$ SDS-polyacrylamide gel. Proteins were transferred onto nitrocellulose membranes by electro-blotting. Membranes were blocked in milk and incubated with primary and secondary antibodies as previously described. ${ }^{1,8}$

The following primary antibodies at the indicated dilutions were used: MEK1, ERK, and phospho-ERK (1:1000; Cell Signaling Technology, Inc., Beverly, MA), and Phospho-MEK1 (Ser217/221) Antibody \#9121; anti-HA probe (1:200; Santa Cruz Biotechnology, Inc., Santa Cruz, CA); anti-MASH1 (mammalian ASH1) to detect ASCL1 (1:1000; Pharmingen); anti-CgA(1:1000; Zymed Laboratories
Inc.); and antiglyceraldehyde-3-phosphate dehydrogenase (GAPDH; 1:10,000; Trevigen, Inc., Gaithersburg, MD). Primary antibody incubations were kept overnight at $4^{\circ} \mathrm{C}$.

The membranes were then washed with wash buffer (phosphatebuffered saline and 1\% Tween-20) and incubated with a 1:2000 dilution of goat anti-rabbit (for MEK1, CgA, and GAPDH) or goat anti-mouse (for MASH1) secondary antibody coupled with horseradish peroxidase (Cell signaling technology). The membranes were again washed with wash buffer and developed with ImmunStar (Bio-Rad) for phospho-MEK1/2, MEK1, CgA, and GAPDH or with Super Signal West Femto chemiluminescent substrate (Pierce) for MASH1according to the manufacturers' directions and the bands were visualized using $\mathrm{X}$-ray films.

\section{MTC morphology}

Morphology of the treated as well as control TT-MEK cells were observed under a light microscope.

\section{Results}

\section{Creation of doxycycline inducible TT-MEK cells}

We successfully created doxycycline inducible TT-MEK cell lines through the sequential transfection method described above.

\section{Dose-dependent functional MEK expression in TT- MEK cells}

To determine the inducibility of MEK by doxycycline in TT-MEK cells, we carried out Western blot analysis after treatment with various concentrations of doxycycline $(0$ to $1 \mu \mathrm{g} / \mathrm{ml})$. As shown in Figure 1, there was no detectable phospho ERK protein in TT-MEK cells in the absence of doxycycline. However, treatment of TT-MEK cells with doxycycline led to a reproducible induction of phospho ERK protein. Notably, the amount of phospho ERK protein was proportional to the dose of doxycycline.

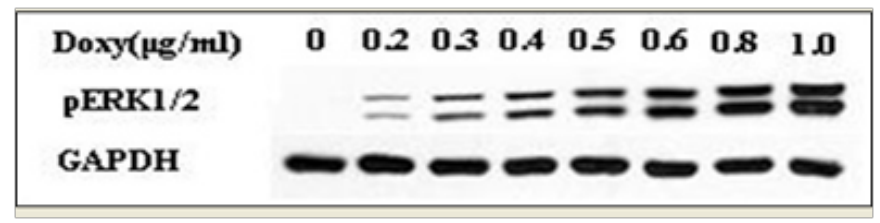

Figure I Dose-dependent activation of ERKI/2 is dependent on the amount of two days doxycycline treatment.

Treatment of TT-MEK cells with doxycycline led to an induction of MEK protein which is associated with activation of ERKI/2 in a dose-dependent manner. Western analysis of protein lysates after 2 days of treatment with doxycycline.

\section{Persistence of MEK induction/ MEK signaling in MTC cells decreases NET markers levels}

When using inducible cell models, it is important to make sure that the model present truly induced cells and to understand the pattern on the inducement. In order to test the effect of induction, TT-MEK cells were treated with and without doxycycline $(0.2,0.5$ and $1 \mu \mathrm{g} / \mathrm{ml})$ up to 4 days. Every 2 days, cell extracts were prepared and analyzed for phosphor ERK, CgA, and ASCL1 protein levels. As shown in Figure 2, phosphor ERK protein was detectable only in the presence of doxycycline, and the induction stayed stable over the 4 days. Cell lysates from TT-MEK cells treated with varying levels of doxycycline 
$(0-1 \mu \mathrm{g} / \mathrm{ml})$ over 2 and 4 days were analyzed for changes in NET marker production. We have shown that an increase in phosphor ERK protein leads to dose-dependent reduction in expression of both ASCL1 \& CgA. The reduction is more with four days of treatment (Figure 3A) (Figure 3B).

\section{MTC morphology changes}

There was a striking morphological change in cells with increased activation of MEK. Cells treated with doxycycline showed a noticeable rounded morphology with loss of cell membrane spread, which is similar to morphologic changes seen with raf-1 activation (Figure 4).

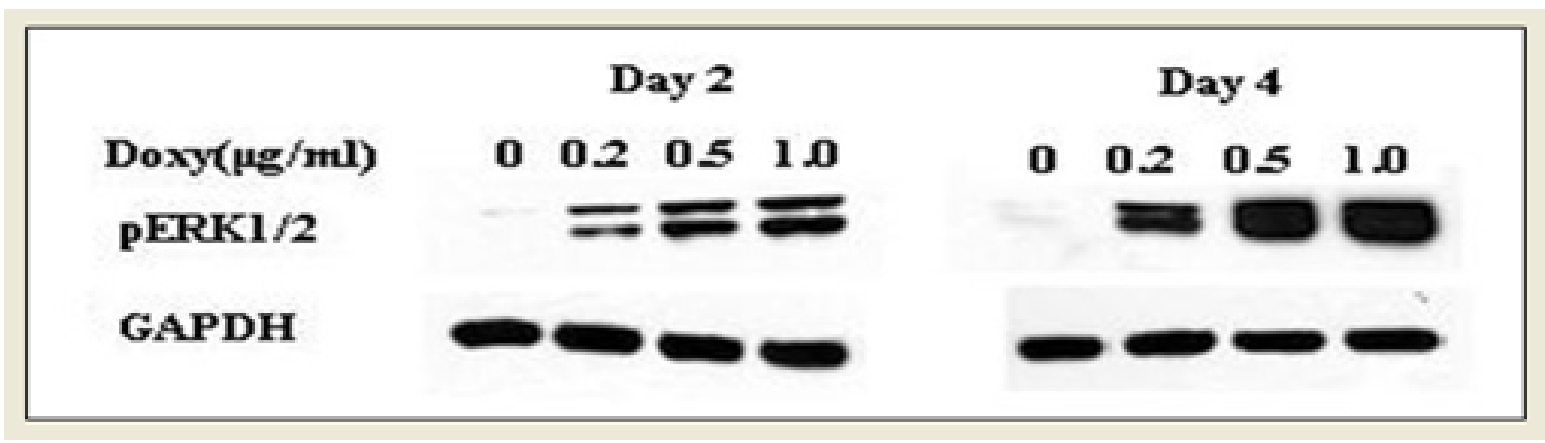

Figure 2 Dose-dependent activation of ERKI/2 is dependent on the amount of four days doxycycline treatment.

Treatment of TT-MEK cells with doxycycline led to an induction of MEK protein which is associated with activation of ERKI/2 in a dose-dependent manner. Western analysis after 4 days of treatment with doxycycline.
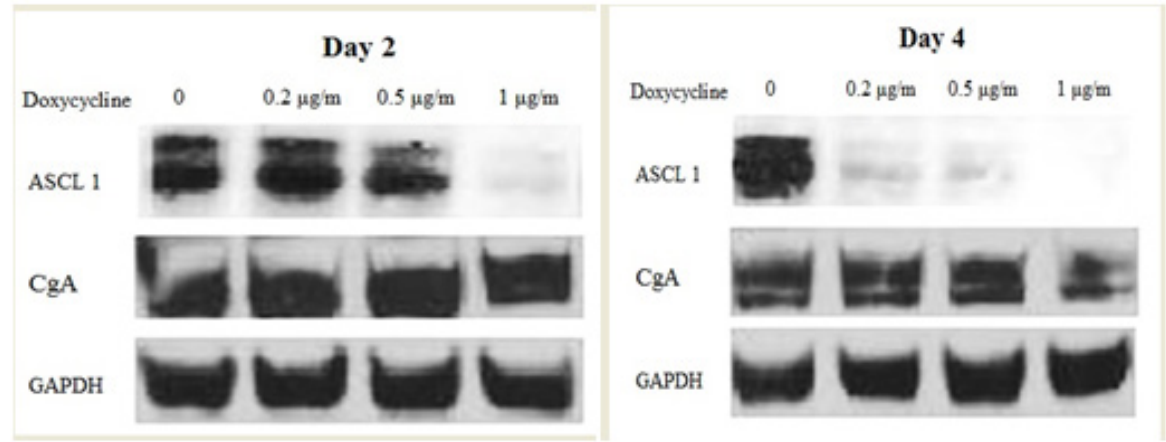

Figure 3 MEK activation reduces the levels of NE markers ASCLI and CgA.The levels of NE marker production were reduced with increasing amounts of MEK activation. GAPDH was used as a protein loading constant. A) Western analysis of protein lysates after 2 days of treatment with doxycycline.

B) Western analysis after 4 days of treatment with doxycycline.

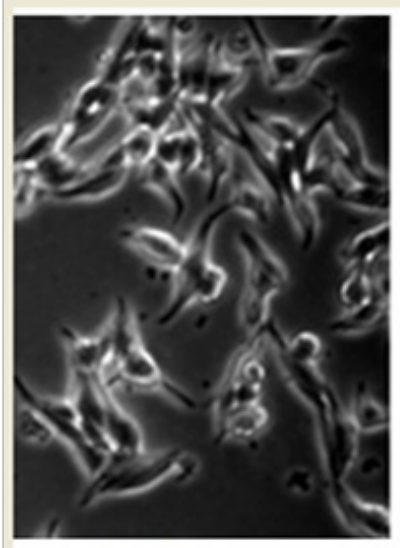

Control

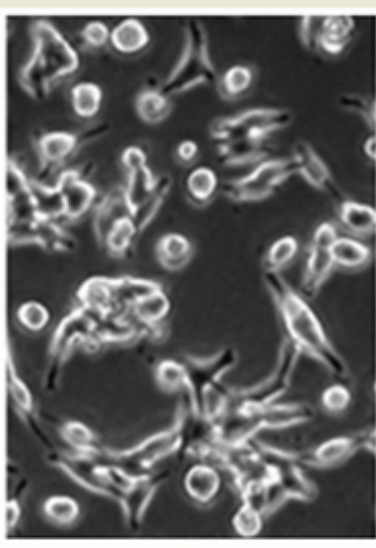

Doxycycline $0.5 \mu g m 1$

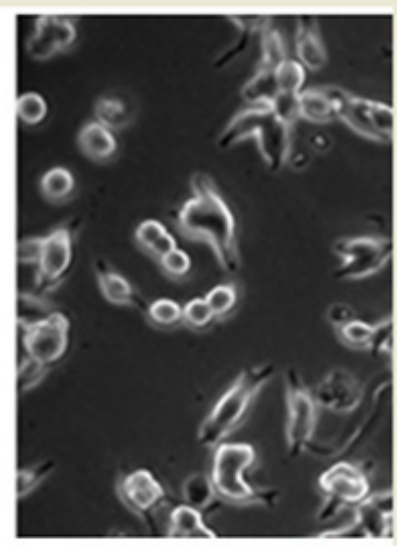

Doxycycline $1 \mu \mathrm{g} / \mathrm{ml}$

Figure 4 MEK activation changes morphology of the TT cells.

There was a striking morphologic change in TT-MEK cells treated with increasing doses of doxycycline. The higher doses, which correspond with higher MEK induction, demonstrated more rounded, larger cells with activation of MEK. 


\section{Discussion}

Medullary thyroid cancer is an indolent neuroendocrine tumor that frequently presents with disseminated disease. While locally advanced tumor in the neck and mediastinum can be treated with surgery either for palliative or curative intent, distant metastases are not often amenable to operative excision. Metastatic MTC has a similarly poor response to radiotherapy and chemotherapy. Viable therapeutic options for distant metastatic disease are still absent. Survival rates after discovery of distant metastasis has been around $25 \%$ at 5 years and $10 \%$ after 10 years, though this has more recently been improved due to an earlier discovery of the malignancy. ${ }^{12,13}$ However, new molecular targets have expedited the oncologic research towards its goal to develop therapeutic agents effective in disseminated disease. ${ }^{14,15}$

Discovery of new therapeutic targets is now possible for MTC. Thus far, these potential targets include kinase inhibitors (RET/ PTC) or signaling kinases such as vascular endothelial growth factor receptor (VEGFR), with a growing number of studies involving the molecular pathways involved in thyroid cancer malignancy. ${ }^{14-21}$ Ras, a $\mathrm{G}$ protein, is an initiator of the $\mathrm{Ras} / \mathrm{RAF} /$ mitogen-activated protein kinase (MEK)/extracellular signal-regulated kinase (ERK) pathway. Phosphorylation of GDP activates Ras that in turn activates Raf. Raf comprises of three cytosolic kinases of which Raf- 1 is more imperative among three for aspects of cell differentiation. ${ }^{1}$

Once Raf activation is initiated, it further initiates MEK and ERK, which are salient in cell differentiation, growth and endurance. ${ }^{5,22} \mathrm{We}$ have shown recently that expression of estradiol-inducible active rafl in pancreatic carcinoid BON cells and MTC cells leads to growth inhibition and reduction in NE hormone production. ${ }^{4}$ However, the role of MEK signaling in MTC cells is not known. Despite the importance of the MEK signaling pathway in cell fate determination, the role of the MEK signaling pathway in MTC has, until now, not been described.

The role of MEK in the field of oncology is of great interest because of its dual nature. It may act as tumor promoter or tumor suppressor depending on the cell milieu. ${ }^{23}$ One study suggests that the MEK signaling pathway is vital in the regulation of MTC phenotype. ${ }^{24}$ In the current work, activation of MEK by doxycycline induction led to an increase in functional MEK protein production in a dosedependent way. Our results depict that continuous MEK activation in TT-MEK cells reduced the levels of CgA and ASCL1. Notably, this suppression was dependent on the levels of the MEK protein present. These findings provide the first documentation of the role of MEK signaling as a tumor marker suppressor in MTC cells.

Activation of the Rafl signaling pathway has been shown to inhibit growth in SCLC, MTC, and carcinoid cells in vitro, ${ }^{6,25,26}$ and we showed that doxycycline-induced MEK expression also led to inhibition of cell growth in MTC cells. Taken together, these results suggest that components of the MEK signaling pathway tightly regulate the MTC phenotype. Raf- 1 activation in MTC cells also leads to significant reductions in the production of tumor markers $\mathrm{CgA}$ and ASCL1. The findings of this study demonstrate a reduction of ASCL1 and $\mathrm{CgA}$ with MEK activation. At the same time, we observed similar morphological changes with both raf-1 activation and MEK activation in MTC cells, where MTC cells with either of these proteins activated tend to have decreased horizontal spread and appear rounder when compared with MTC cells without raf-1 or MEK activation.

\section{Conclusion}

This study illustrates that activation of MEK decreases neuroendocrine (NE) marker production in MTC cells. We demonstrate, for the first time, that the over expression of MEK in MTC cells results in similar effects of raf-1 pathway activation. Thus, MEK activation may serve as a molecular target in therapeutic strategies for patients with disseminated MTC.

\section{Funding}

a) American Cancer Society Research Scholars Grant.

b) American Cancer Society MEN2 Thyroid Cancer Professorship.

c) Ministry of higher education in Saudi Arabia, and KFSH\&RC, Riyadh, Saudi Arabia for fellowship and financial support (Amal Alhefdhi).

\section{Acknowledgments}

None.

\section{Conflicts of interest}

The author declares there is no conflict of interest.

\section{References}

1. Kunnimalaiyaan M, Vaccaro AM, Ndiaye MA, et al. Overexpression of the NOTCH1 intracellular domain inhibits cell proliferation and alters the neuroendocrine phenotype of medullary thyroid cancer cells. J Biol Chem. 2006;281(52):39819-39830.

2. Stratakis CA, Ball DW. A concise genetic and clinical guide to multiple endocrine neoplasias and related syndromes. $J$ Pediatr Endocrinol Metab. 2000;13(5):457-465.

3. Minakshi M, Arghya A, Samik C, et al. Targeting RET to induce medullary thyroid cancer cell apoptosis: an antagonistic interplay between PI3K/Akt and p38MAPK/ caspase-8 pathways. Apoptosis. 2013;18(5):589-604.

4. Nakakura EK, Sriuranpong VR, Kunnimalaiyaan M, et al. Regulation of neuroendocrine differentiation in gastrointestinal carcinoid tumor cells by notch signaling. J Clin Endocrinol Metab. 2005;90(7):4350-4356.

5. Park JI, Strock CJ, Ball DW, et al. The Ras/Raf/MEK/extracellular signal-regulated kinase pathway induces autocrine-paracrine growth inhibition via the leukemia inhibitory factor/JAK/STAT pathway. Mol Cell Biol. 2003;23(2):543-554.

6. Torino F, Paragliola RM, Barnabei A, et al. Medullary thyroid cancer: a promising model for targeted therapy. Curr Mol Med. 2010;10(7):608625.

7. Antonell A, Fallahi P, Ferrari SM, et al. RET TKI: Potential Role in Thyroid Cancers. Curr Oncol Rep. 2012;14(2):97-104.

8. Kunnimalaiyaan $\mathrm{M}$, Chen $\mathrm{H}$. The Raf-1 pathway: a molecular target for treatment of select neuroendocrine tumors? Anticancer Drugs. 2006;17(2):139-142.

9. Dhillon A, Kolch W. Oncogenic B-Raf mutations: crystal clear at last. Cancer Cell. 2004;5(4):303-304.

10. Oishi K, Kamakura S, Isazawa Y, et al. Notch promotes survival of neural precursor cells via mechanisms distinct from those regulating neurogenesis. Dev Biol. 2004;276(1):172-184.

11. Zarebczan B, Chen H. Signaling Mechanisms in Neuroendocrine Tumors as Targets for Therapy. Endocrinol Metab Clin North Am. 2010;39(4):801-810. 
12. Sippel RS, Carpenter JE, Kunnimalaiyaan M, et al. The role of human achaete-scute homolog-1 in medullary thyroid cancer cells. Surgery. 2003;134(6):866-871.

13. Sippel RS, Carpenter JE, Kunnimalaiyaan M, et al. Raf-1 activation suppresses neuroendocrine marker and hormone levels in human gastrointestinal carcinoid cells. Am J Physiol Gastrointest Liver Physiol. 2003;285(2):G245-G254.

14. Stjepanovic N, Capdevila J. Multikinase inhibitors in the treatment of thyroid cancer: specific role of lenvatinib. Biologics. 2004;8:129-39.

15. Grande E, Diez JJ, Zafon C, et al. Thyroid cancer: molecular aspects and new therapeutic strategies. J Thyroid Res. 2012;2012:847108.

16. Wells SA, Robinson BG, Gagel RF, et al. Vandetanib in patients with locally advanced or metastatic medullary thyroid cancer: a randomized, double-blind Phase III trial. J Clin Oncol. 2012;30(2):134-141.

17. Elisei R, Schlumberger MJ, Müller SP, et al. Cabozantinib in progressive medullary thyroid cancer. J Clin Oncol. 2013;31(29):3639-3646.

18. Karras S, Anagnostis P, Krassas GE. Vandetanib for the treatment of thyroid cancer: an update. Expert Opin Drug Metab Toxicol. 2014;10(3):469-481.

19. Motylewska E, Lawnicka H, Kowalewicz-Kulbat M, et al. Interferon alpha and rapamycin inhibit the growth of carcinoid and medullary thyroid cancer in vitro. Pharmacol Rep. 2014;66(4):624-629.

20. Durante C, Paciaroni A, Plasmati K, et al. Vandetanib: opening a new treatment practice in advanced medullary thyroid carcinoma. Endocrine. 2013;44(2):334-342.
21. Nagilla M, Brown RL, Cohen EE. Cabozantinib for the treatment of advanced medullary thyroid cancer. Adv Ther. 2012;29(11):925-934.

22. Van Gompel JJ, Kunnimalaiyaan M, Holen K, et al. ZM336372, a Raf1 activator, suppresses growth and neuroendocrine hormone levels in carcinoid tumor cells. Mol Cancer Ther. 2005;4(6):910-917.

23. Adams DG, Coffee RL, Zhang $\mathrm{H}$, et al. Positive Regulation of Raf1-MEK1/2-ERK1/2 Signaling by Protein Serine/Threonine Phosphatase 2A Holoenzymes. The Journal of Biological Chemistry. 2006;280(52):42644-42654.

24. Kloos RT, Eng C, Evans DB, et al. Medullary thyroid cancer: management guidelines of the American Thyroid Association. Thyroid. 2009;19(6):565-612.

25. Chen H, Kunnimalaiyaan M, Van Gompel J. Medullary thyroid cancer: the functions of raf-1 and human achaete-scute homologue-1. Thyroid. 2005;15(6):511-521.

26. Kunnimalaiyaan M, Traeger K, Chen H. Conservation of the Notch1 signaling pathway in gastrointestinal carcinoid cells. Am J Physiol Gastrointest Liver Physiol. 2005;289(4):G636-G642. 